\title{
NATURE OF THE VENUS CLOUDS AS DERIVED FROM
}

\section{THEIR POLARIZATION}

\author{
JAMES E. HANSEN \\ Goddard Institute for Space Studies, New York, N.Y., U.S.A. \\ and \\ J. W. HOVENIER \\ Vrije Universiteit, Amsterdam, The Netherlands
}

\begin{abstract}
The linear polarization of sunlight reflected by Venus is analyzed by comparing observations with extensive multiple scattering computations. The analysis establishes that Venus is veiled by a cloud or haze layer of particles which have a narrow size distribution with a mean radius $\sim 1 \mu$. The refractive index of the particles is $1.44 \pm 0.015$ at $\lambda=0.55 \mu$ with a small normal dispersion, the refractive index decreasing from the ultraviolet toward the infrared. The particles exist at a high level in the atmosphere, with the optical thickness unity occurring where the pressure is about $50 \mathrm{mb}$.

The particle properties deduced from the polarization eliminate all but one of the cloud compositions which have been proposed for Venus. A concentrated solution of sulfuric acid $\left(\mathrm{H}_{2} \mathrm{SO}_{4}-\mathrm{H}_{2} \mathrm{O}\right)$ provides good agreement with the polarization data.
\end{abstract}

Polarization observations at present provide the most sensitive means for determination of the cloud particle characteristics on Venus. In the analysis of the polarization it is essential that the effects of multiple scattering be accurately accounted for, as has been demonstrated by Hansen and Hovenier (1971). Thus in order to help extract the available information on the cloud particles we have made a series of multiple scattering computations and compared them with observations of Venus made by Lyot (1929), Kuiper (1957), Marin (1965), Dollfus (1966), Coffeen and Gehrels (1969), Dollfus and Coffeen (1970), Forbes (1971), and Veverka (1971). Our computations are much more extensive than those of Hansen and Arking (1971); we have included many observations not employed by Hansen and Arking and we have analyzed observations at all available wavelengths. In addition we have related the derived cloud particle refractive index and its dispersion to the proposed cloud compositions for Venus. A paper giving a detailed documentation of our method of analysis is in preparation and will be submitted for publication in the Journal of the Atmospheric Sciences.

Our computations for the degree of linear polarization involve three steps. First the well-known Mie theory is used to compute the single scattering properties of a size distribution of spherical particles. We have found that in practice it is usually possible to describe the effect of the size distribution by two parameters which describe the mean particle size and the width of the size distribution. The second part of the computations is for multiple scattering by a homogeneous plane-parallel atmosphere. This we accomplish with the doubling method which was developed for the case of polarized light by Hansen (1971) and Hovenier (1971). Finally we integrate the multiple scattering results over the planetary disk, assuming a spherical but locally-plane- 
parallel atmosphere. The basic method of integration is the same as that described by Horak (1950). For the limited number of observations referring to a small area on the planet we of course used the computations without integration over the disk.

Several prominent features in the theoretical polarization depend on the spherical shape of the particles. These include the 'rainbow', the 'glory' and a feature of 'anomalous diffraction'. The existence of all these features in the observed polarization of Venus, in close agreement with the theory, is sufficient to prove that the particles in the visible clouds of Venus are in fact spherical. In addition we find that the effective radius for the particle size distribution, defined as

$$
r_{\text {eff }}=\frac{\int_{0}^{\infty} r \pi r^{2} n(r) \mathrm{d} r}{\int_{0}^{\infty} \pi r^{2} n(r) \mathrm{d} r},
$$

is $1.05 \pm 0.1 \mu$, and that the effective variance, defined as

$$
v_{\text {eff }}=\frac{\int_{0}^{\infty}\left(r-r_{\text {eff }}\right)^{2} \pi r^{2} n(r) \mathrm{d} r}{r_{\text {eff }}^{2} \int_{0}^{\infty} \pi r^{2} n(r) \mathrm{d} r},
$$

is $0.07 \pm 0.02 . n(r) \mathrm{d} r$ is the number of particles with radius between $r$ and $r+\mathrm{d} r$. The spherical particle shape and the narrow size distribution strongly suggest that the cloud particles on Venus are liquid.

The particle refractive index is perhaps the most important information which we extract from the polarization. For this we obtain $n_{r}(\lambda=0.55 \mu)=1.44 \pm 0.015$, where $n_{r}$ decreases slightly with increasing wavelength, from $n_{r}(\lambda=0.365 \mu)=1.46 \pm 0.015$ to $n_{r}(\lambda=0.99 \mu)=1.43 \pm 0.015$. In order to compare these values with laboratory values of $n_{r}$ for proposed cloud constituents we must be able to interpolate (or extrapolate) the laboratory values in wavelength and temperature. We handle this problem by using two formulae of classical physics (cf. Born and Wolf, 1959): the Lorentz-Lorenz equation, which yields the dependence of the refractive index on temperature, and a dispersion formula for classical oscillators, which yields the dependence of the refractive index on wavelength. We are able to demonstrate that this procedure is satisfactory for substances which do not have a strong absorption band in the visible part of the spectrum.

Of all the cloud compositions which have been proposed in the literature for Venus, we find that only a concentrated solution of sulfuric acid is in agreement with the polarization data. Other evidence in support of sulfuric acid on Venus has been presented by Young (1973) and Sill (1972). In particular we find that the refractive indices of hydrochloric acid solutions and carbon suboxide are definitely not compatible with the values of $n_{r}$ for the Venus cloud particles. These materials had previously been considered as candidates for the cloud composition, in large part because their refractive indices were in the general range of values indicated by the polarization. 
Finally, the polarization also indicates that the cloud particles occur high in the atmosphere of Venus, with the optical depth unity being at a pressure $\sim 50 \mathrm{mb}$. This conclusion, unlike those for the particle shape, size distribution and refractive index, is not independent of the homogeneous model atmosphere which we employ. However we estimate that the uncertainty in the value $50 \mathrm{mb}$ is at most $\sim 25 \mathrm{mb}$.

It is notable that there are some marked similarities between the particles in the atmosphere of Venus and the aerosol layer on the Earth at an altitude $\sim 20 \mathrm{~km}$ (the Junge layer), as we have previously pointed out (cf. Hunten, 1971). The Junge layer is at the pressure level $\sim 50 \mathrm{mb}$ and the particle size distribution is apparently very narrow (Friend, 1966; Mossop, 1965), corresponding to $v_{\text {eff }}=0.06-0.08$. On the other hand the particles in the Junge layer are smaller $\left(r_{\mathrm{eff}}=0.3-0.4 \mu\right.$; Friend, 1966) and have a smaller optical thickness $\left(\sim 2 \times 10^{-2}\right.$; Elterman et al., 1973) than the particles on Venus. Nevertheless the above similarities, together with the fact that the Junge layer contains a large fraction (perhaps more than 50\%; Rosen, 1971, Lazrus et al., 1971) of sulfuric acid, offer additional support for the conclusion that the particles in the visible clouds of Venus are composed of strong sulfuric acid solutions.

\section{Acknowledgements}

J.E.H. was supported during the course of part of this work by NASA Grant 33008-012 through Columbia University. J.W.H. was supported by the Netherlands Organization for the Advancement of Pure Research (Z.W.O.) during a one year stay at the Institute for Space Studies in 1970-71 when this work was originated.

\section{References}

Born, M. and Wolf, E.: 1959, Principles of Optics, Pergamon, London, p. 86.

Coffeen, D. L. and Gehrels, T.: 1969, Astron. J. 74, 433.

Dollfus, A.: 1966, Contribution au Colloque Caltech-JPL sur la lune et les planètes: Venus. JPL

Tech. Memo. No. 33-226, 187.

Dollfus, A. and Coffeen, D. L.: 1970, Astron. Astrophys. 8, 251.

Elterman, L., Toolin, R. B., and Essex, J. D.: 1973, Appl. Opt. 12, 330.

Forbes, F. F.: 1971, Astrophys. J. 165, L21.

Friend, J. P.: 1966, Tellus 18, 465.

Hansen, J. E.: 1971, J. Atmospheric Sci. 28, 120.

Hansen, J. E. and Arking, A.: 1971, Science 171, 669.

Hansen, J. E. and Hovenier, J. W.: 1971, J. Quant. Spectrosc. Radiat. Transfer 11, 809.

Horak, H. G.: 1950, Astrophys. J. 112, 445.

Hovenier, J. W.: 1971, Astron. Astrophys. 13, 7.

Hunten, D. M.: 1971, Space Sci. Rev. 12, 539.

Kuiper, G. P.: 1957, in M. Zelikoff (ed.), Threshold of Space, Pergamon, New York, p. 78.

Lazrus, A. L., Gandrud, B., and Cadle, R. D.: 1971, J. Geophys. Res. 76, 8083.

Lyot, B.: 1929, Ann. Obs. Paris (Meudon) 8, [available in English as NASA TT F-187, 1964].

Marin, M.: 1965, Rev. Optique 44, 115.

Mossop, S. C.: 1965, Geochim. Cosmochim. Acta 29, 201.

Rosen, J. M.: 1971, J. Appl. Meteorol. 10, 1044.

Sill, G. T.: 1972, Comm. Lunar Planet. Lab., No. 171, 191-198.

Veverka, J.: 1971, Icarus 14, 282.

Young, A. T.: 1973, Icarus 18, 564. 


\section{DISCUSSION}

Icke: The straight-line relationships which you found are determined by the slope and intercept of the lines. Which physical properties of the molecules determine these two parameters?

Hovenier: These parameters are essentially determined by the mean resonance frequency and by the mean oscillator strength of the molecules.

Van de Hulst: There seems to exist a very fundamental relation: 'much money $\ll$ good ideas'! 